\title{
How bread can save the world
}

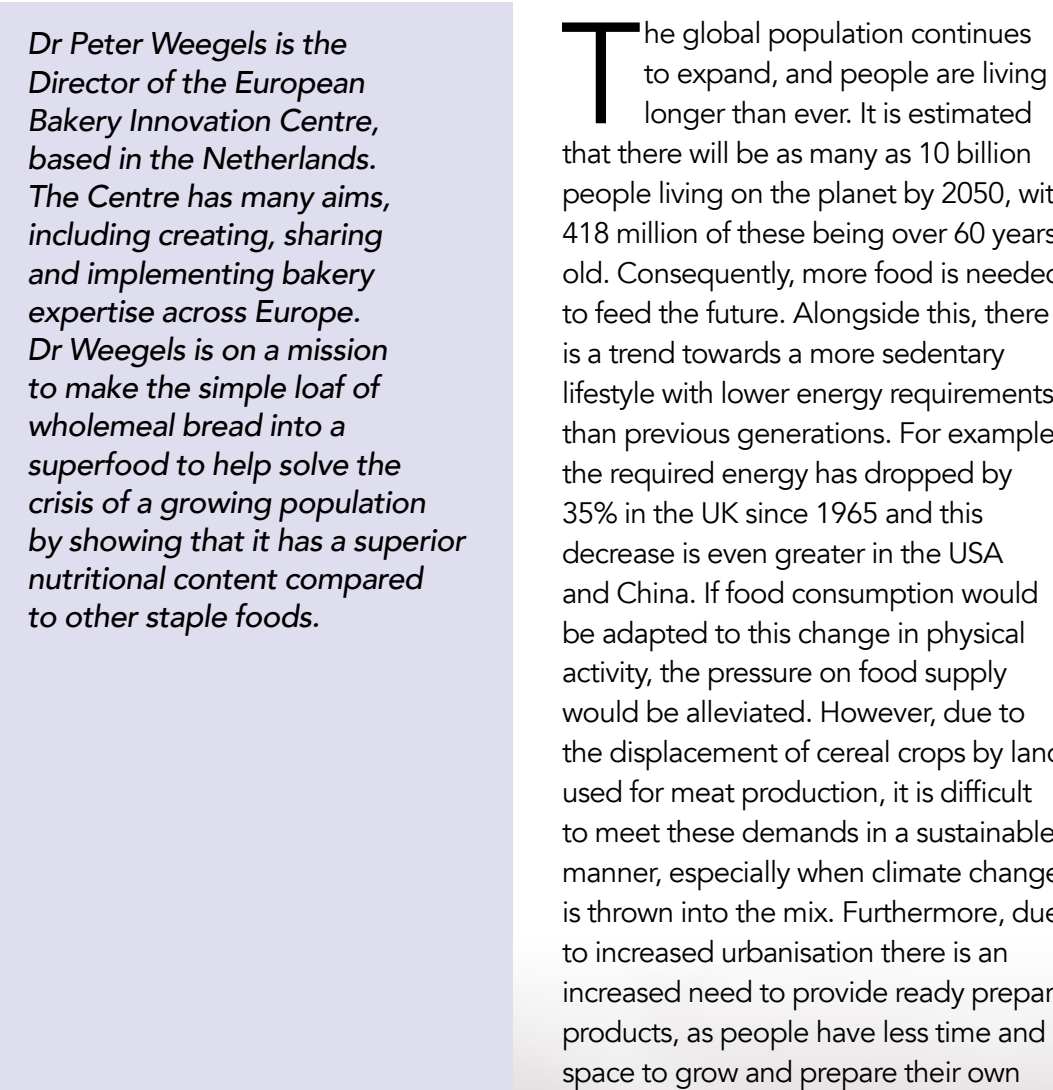

Dr Peter Weegels is the Director of the European Bakery Innovation Centre, based in the Netherlands. The Centre has many aims including creating, sharing and implementing bakery expertise across Europe. to make the simple loaf of wholemeal bread into a superfood to help solve the crisis of a growing population by showing that it has a superio nutritional content compared to other staple foods. to increased urbanisation there is an products, as people have less time and space to grow and prepare their own and China. If food consump tio activity, the pressure on food used for meat production, it to meet these demands in

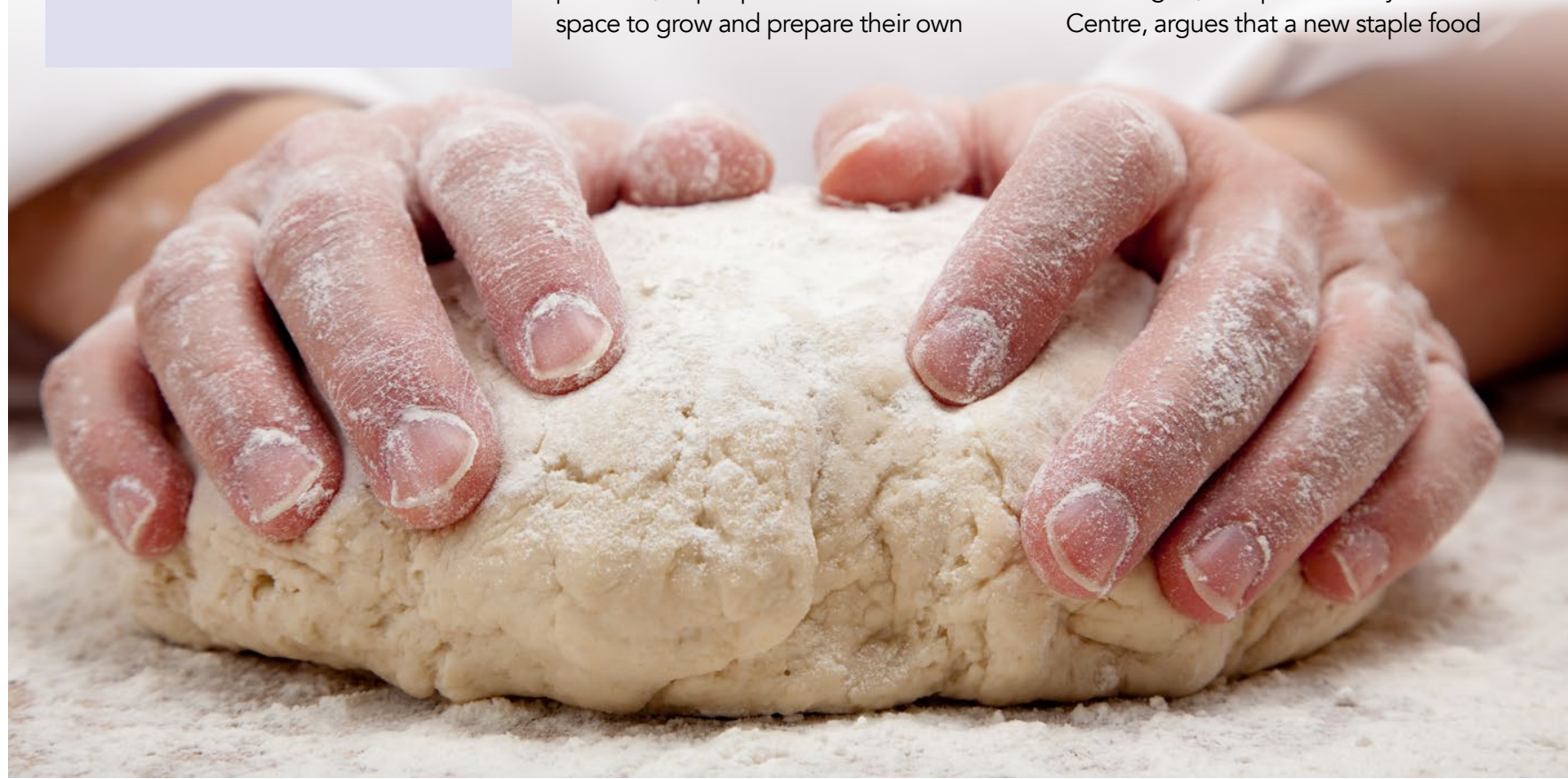

food. Finally, although a decrease in food intake should be made due to a more sedentary lifestyle, this rarely occurs, and in combination with easily accessible and abundance of food round the clock, they contribute to an increase in obesity. Despite overconsumplion of calonies this is often associated with insuficions

Next to the previous challenges and at the other side of the spectrum in less effluent populations malnutrition from insufficient foods supply is still a large problem. Both extremes are becoming more prevalent and the gap between the lowest and highest energy intakes is growing. Indeed, it is estimated that around 4 million people are at risk of malnutrition, therefore a more nutritious and secure food supply is required to Weegels is working toward.

\section{BREAKING BPEAD} combat this risk and it is this that Dr Peter

Weegels, European Bakery Innovation

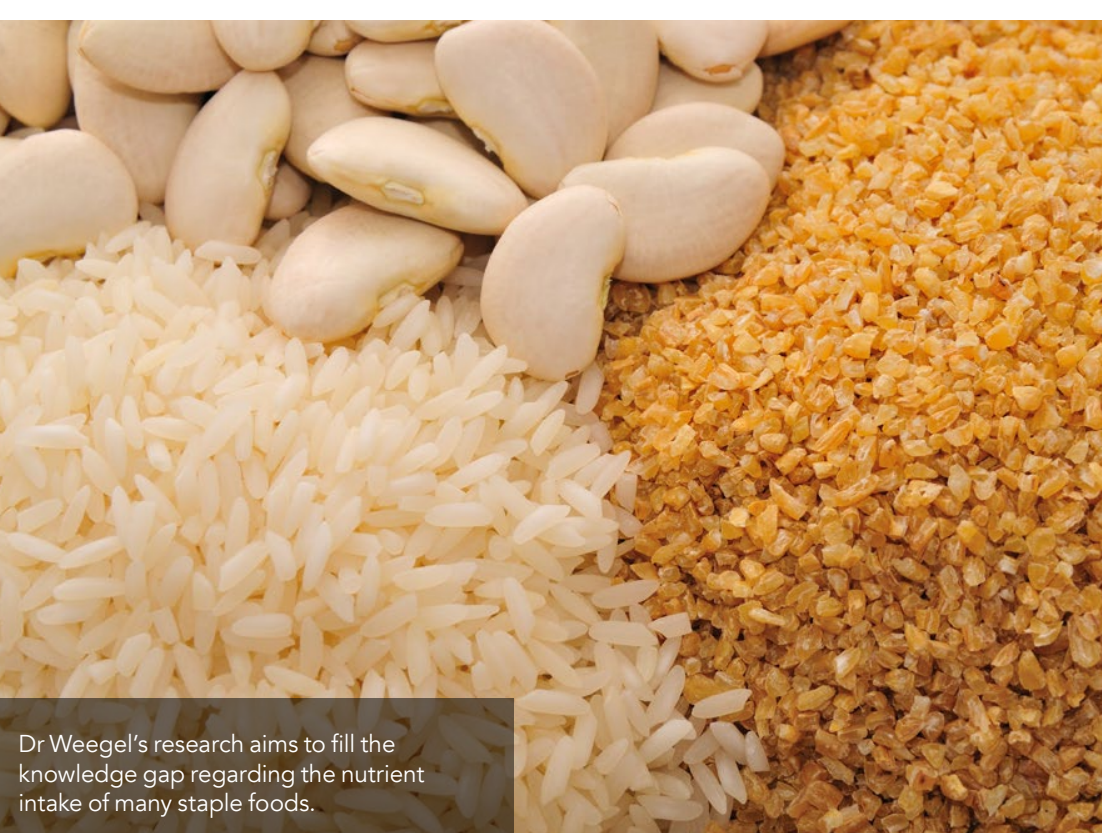

is needed in the form of bread, specifically wholemeal be for hof bead, specif based foods, such as cereals, roots and tubers currently contribute significantly to nutrient intake and have an important role in many of the recommended diets, such as the Mediterranean diet. There is an average annual growth rate of $1.7 \% \mathrm{for}$ wheat yields to fulfil the growing demand but this may not be enough to feed the growing population.

However, a comparison of the recommended nutrentintakes from these staple foods is lacking, and this is the knowledge gap that Dr Weegels seeks to fill. There has been much discussion about the best way to carry out nutrition profling. For example, compositional foods, these are usually based on the unprocessed products, rater the in a ready-to-eat form. Factors such as seasonality, soil quality and geographica region can also cause variation in nutrient profile within the same product

Therefore, DrWeegels, who also works in partnership with Wageningen University and Research, the most important university in the Netherland which focuses specifically on food bioprocessing, nutrition and healthy living. decided to undertake this comparison. He selected two detailed nutrition tables, the USA Food Nurition Table and the Dutch Nun Fon Table and calculated the contribution of ready to eat staple

daily reference intakes (DRI) based on US and EU recommendations. The nutrition tables contained average values from multiple sources, providing the most of a particular food. Staple foods were as starchy foods including rice, a

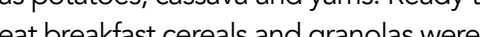
not included, as these are not regarded staple foods. As many bread products as now enriched with vitamins and minerals in order to address population deficiencies, the non-enriched levels of these micronutrients were estimated, to exclude bias from fortification. To obtain the equivalent nutritional value of
wholemeal bread, two to three times more teff
or quinoa would need to be consumed.

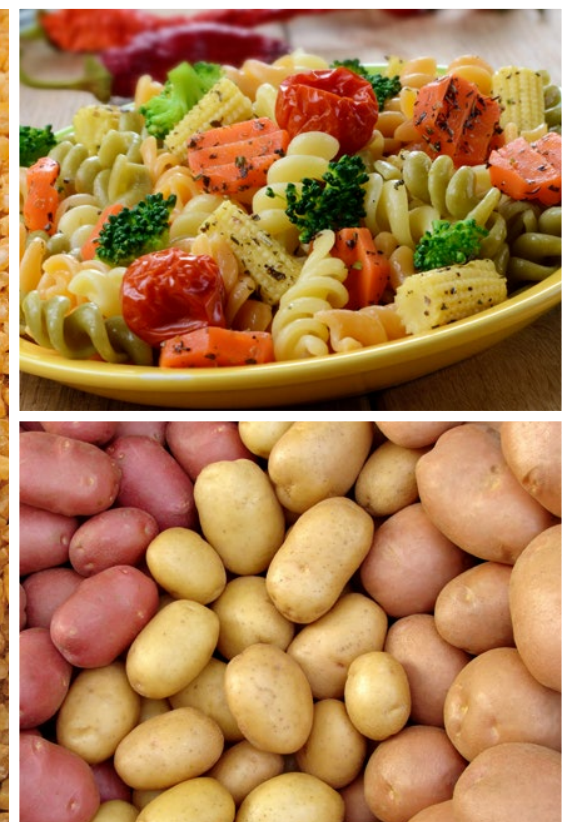

There are different DRI and RDA values available for men, women and childre t different ages, as different subpopulations have varying requirements and Dr Weegels selected the value for adult men and an average body weight of $70 \mathrm{~kg}$ was used to calculate protein

Dr Weegels, European Bakery Innovation Centre, argues that a new staple food is needed in the form of bread, specifically improved wholemeal bread.

wheat, maize, pseudocereals such as millet, requirements. The nutrient content was

calculad per $100 \mathrm{~g}$ prepared product, ding a direct comparison.

The comparison done by DrWeegels concluded that the most beneficial providing highest amounts of protein carbohydrate, energy and fibre as well as minerals per 100g. The bread category

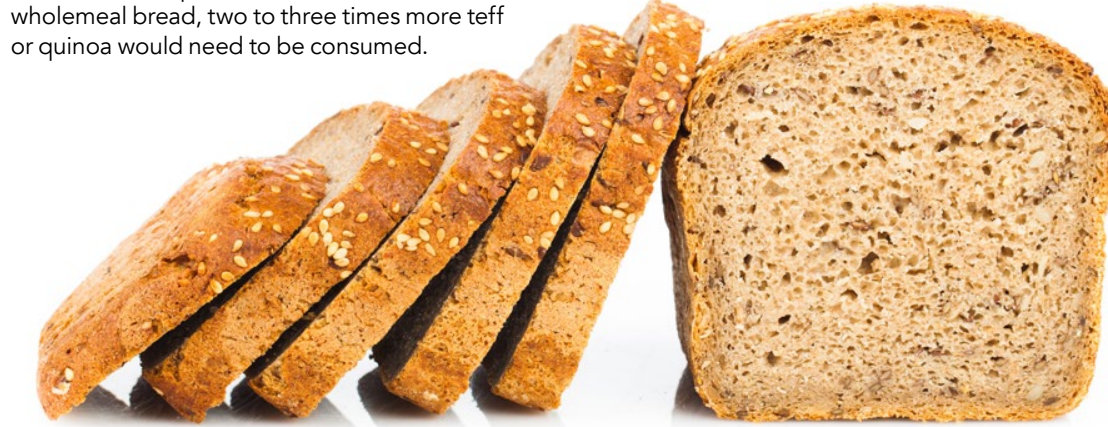


CONTRIBUTION OF STAPLE FOODS TO RDI Macronutrients
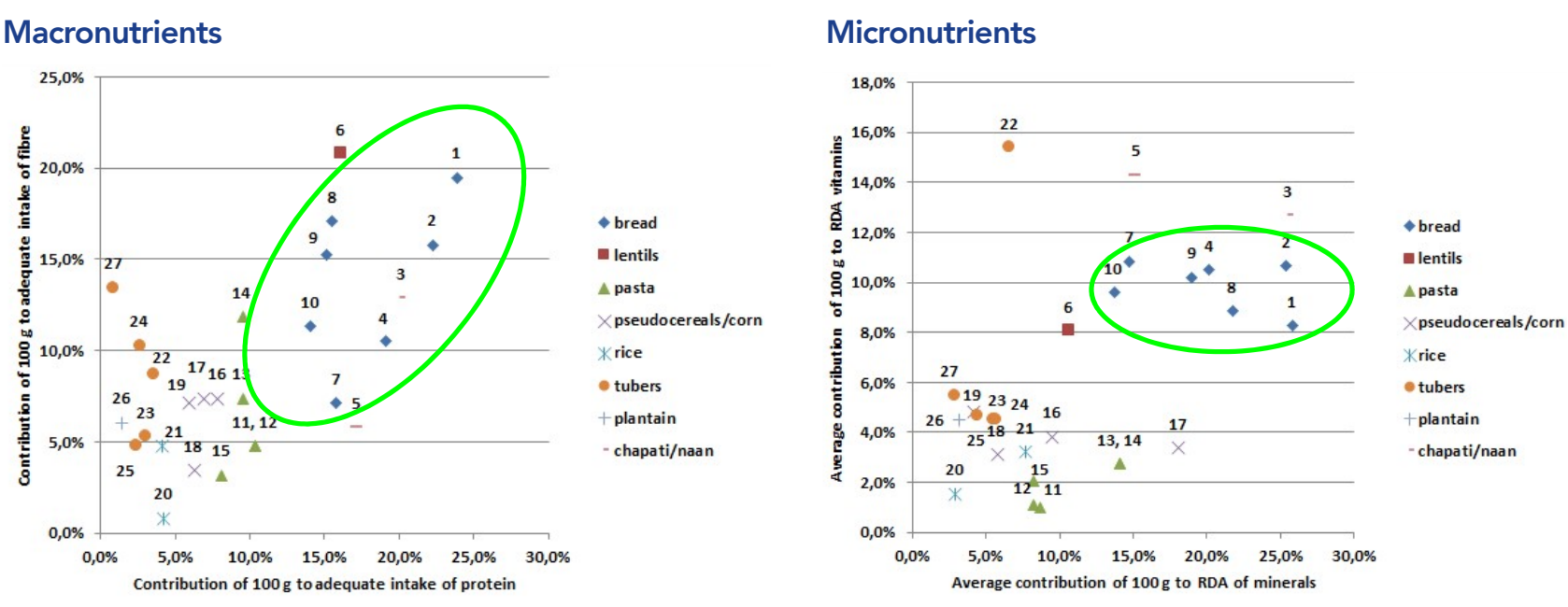

Bread provides the highest amounts of protein, carbohydrate, energy, fibre and minerals per $100 \mathrm{~g}$ compared to other staple foods.

also makes an important contribution coseting dally vitamin needs. In the amount of fibre from wholemeal bread four times the amount of boiled potatoes would need to be consumed It is also challenging to consider the contribution from other staple foods, as they make up just a small part of the overall daily intake. This trend continues when the average daily bread consumption is compared across several countries (including white and wholemeal bread), suggesting that a much larger amount of the other foods would be needed to match the contribution of bread. It would be interesting to look at cost too, as this may be a barrierts the consumption of some staple foods, with avallability of products varying between counties. Despite already been observed over recent years

Wholemeal bread is the often-overlooked staple food with the ability to combat future food availability and nutritional health challenges.

in continents that traditionally do not eat bread as a staple food, like Africa and Asia. Finally, increasing numbers of due to an intolerance or allergy, but also (n) Therefore, more research is needed into gluten free products, alongside a focus on food safety regarding potentia allergens. Next to the significant increas incosts of the altemative diets, adequare thallen and even a helh thotant this is not managed properly.

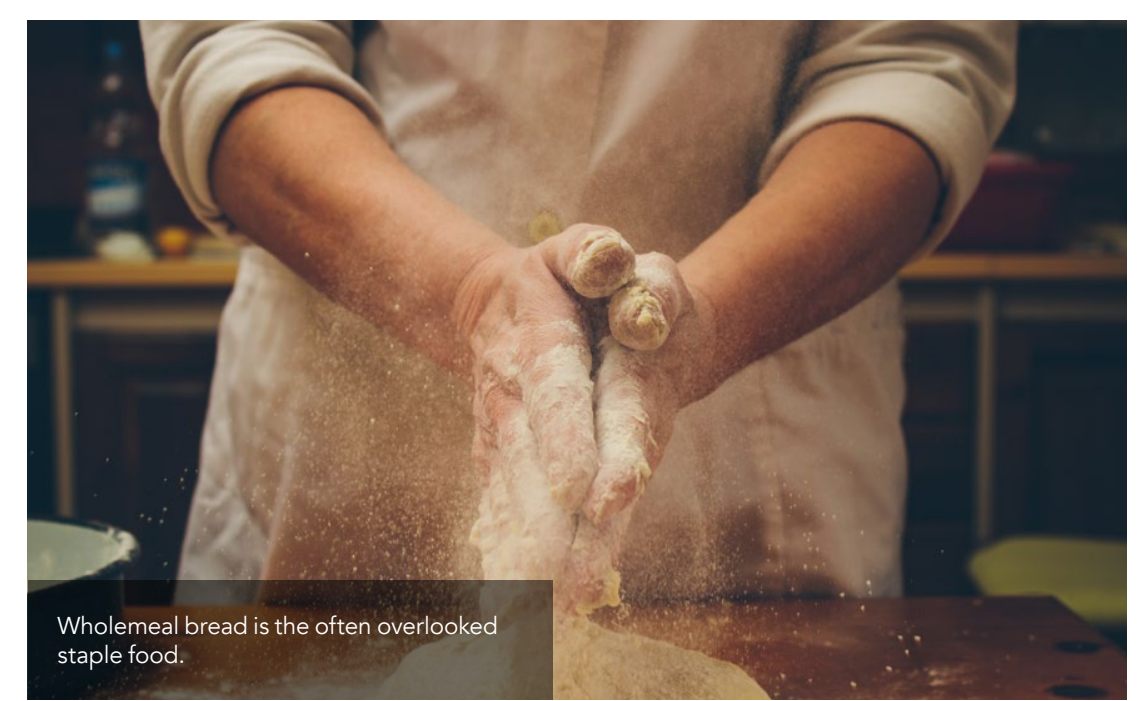

BREAD: THE NEXT SUPERFOOD superfood to the forefront of socia media, perhaps the humbler foods have been overlooked. For example, to obtain the equivalent nutritional value of wholemeal bread, two to three mes more teff or quinoa, or nine times be consusels sprouts would need to be consumed. Dr Weegels points out tha obtaining them Reduced bi savilability of nutrients and the amount found in the food may not always match. In orde to combat this, further research is needed determine the best way to process, or not process, foods to optimise nutrient use by the body.

DrWeegels' study concludes that wholemeal bread is the often overlooked staple food with the ability to combat future food availability and nutrition health challenges, and that future trends should encourage consumption. is convenient to consume and potentially contributes to the intake of many important nutrients. Yet some challenges remain, such as increasing sustainability of the food chain by educing waste, reducing the salt content of bread and improving the digestibility

\section{Behind the Research}

\section{Dr Peter Weegels}

E: peter.weegels@sonneveld.com T: +31651637454 W: www.ebic.info

\section{Research Objectives}

The many aims of The European Bakery Innovation Centre include creating, sharing and implementing bakery expertise across Europe.

\section{Detai}

European Bakery Innovation Centre, Sonneveld Group BV Rietgorsweg 1-3,3356 LJ Papendrecht, the Netherlands Bio

My fundament is to connect soil with spirit for which growth is a prerequisite, i.e. by growing people, business and knowledge. My professional mission is to make Cereal Science work, by developing excellent bakery concepts (awarded three times) and creation and global dissemination of knowledge and expertise (open innovation, organising > 35 international meetings and $>50$ publications)

\section{References}

Weegels, P.L. (2019). The future of bread in view of its contribution to nutrient intake as a starchy staple food. Plant Foods for Human Nut lion. 74(1), pp. 1-9. dol: 10.1007/

\section{Personal Response}

Bread has a very short shelf life, compared to other
food stuffs such as dried lentils which can be stored food stuffs such as dried lentils which can be stored associated with storage, especilly in hot humid or lower socioeconomic countries?

II Although shelf life of bread can be extended to a month or more, shelf life of wheat and flour are substantially or industrial bakers and sell it as fresh as possible. Even in lower socioeconomic countries this is practice, many times with mixing local (pseudo)cereals to support 